\title{
Calorimetric and Rheological Measurements of Three Commercial Thermosetting Prepreg Epoxies
}

\author{
Mitch HaRgis AND BRIAN P. GRADY* \\ School of Chemical, Biological and Materials Engineering \\ University of Oklahoma \\ 100 East Boyd St., Norman, OK 73019, USA \\ LEVEnt AKtas, Krishnasumanth R. BOMiReddy, \\ Steve Howsman And M. Cengiz Altan \\ School of Aerospace and Mechanical Engineering \\ University of Oklahoma \\ 865 Asp Avenue, Norman, OK 73019, USA \\ TOM Rose AND Hans Rose \\ Advanced Processing Technology, 2795 Broce Dr. \\ Norman, OK 73072, USA
}

(Received April 2, 2004)

(Accepted April 11, 2005)

\begin{abstract}
The cure kinetics of three different thermosetting resins are investigated using differential scanning calorimetry and oscillatory shear rheometry. For the latter, two different types of plates are used, smooth plates and grooved plates; the latter are used to improve sample-plate contact. In addition, oscillatory compression rheology is used; however, machine compliance prevents accurate measurements at high conversions. A fractional conversion is defined based on the maximum storage modulus achieved at a given temperature, and is compared to the fractional conversion calculated from enthalpy measurements. As expected, the rates of reaction derived from these fractional conversions are very different for calorimetry and rheometry. However, the rates of reaction using the two types of plates are identical, although the grooved plates give much more reproducible storage moduli. A number of previously used mathematical expressions are employed to fit the calorimetric and rheological data, and the activation energies calculated from these fits are compared.
\end{abstract}

KEY WORDS: epoxy, cure model, differential scanning calorimetry, rheology.

*Author to whom correspondence should be addressed. E-mail: bpgrady@ou.edu

Journal of COMPOSITE MATERIALS, Vol. 40, No. 10/2006 


\section{INTRODUCTION}

Q UANTIFICATION OF A composite's properties during cure is an important industrial problem and has attracted a great deal of interest in the literature. The importance can be viewed in two different ways. First, for a given time-temperature profile, which may be different in different areas of the same finished piece, one would like to be able to predict the properties. The properties of particular interest are typically various mechanical properties, i.e. flexural strength, strain to failure. More precisely, the prediction should be able to predict how the properties vary in use; particularly in relationship to environmental stimulants such as temperature and water. Second, for a given state of the material during curing, one would like to adjust the remaining timetemperature profile to reach a certain target value of some finished product variable or a combination of finished product variables. Our particular interest in this problem is composite repair, where the reproducible control of a time-temperature cycle as found in a typical autoclave of a typical original manufacturer may not be possible. Hence, it is really the second of these issues, control, which is the overall concern of our work in this area.

One of the necessary prerequisities for a good control scheme is a predictive model for various resin properties. Note the shift from composites to resins; in general, the fiber can be considered chemically inert, so translating resin properties to composite properties is typically straightforward if the volume fraction and orientation of the fibers are known. The important fundamental material properties during curing of the resin include thermal conductivity, viscosity, heat capacity, and the interfacial tension of the resin. ${ }^{1}$ All the four properties must be known at a given instant in time for a given time-temperature history. Further, in almost all real situations, these properties may vary spatially as well because of the variations in the time-temperature history for different parts of the sample. Of thermal conductivity, heat capacity, viscosity, and interfacial tension, the first two can be determined by a fairly simple combination of the pure component properties. ${ }^{2}$ The interfacial tension, or more precisely the ability of the resin to completely wet the solid, is typically assumed to be irrelevant, i.e., in practice the solid and/or the resin is adjusted so that the solid is completely wet by the resin. A great deal of formulation expertise goes into making this assumption a reality; difficulties in measurement typically preclude measuring this quantity in a reacting system. The fourth variable, viscosity, varies substantially with the fractional conversion and temperature in a non-simple way, hence many measurements of fundamental material properties have focussed on the viscosity.

In a control sense, the determination of a material property at a given time in a cure cycle usually requires the determination of the fractional conversion first, and then a table or mathematical formula is consulted to determine the material property. The table or mathematical formula is typically generated in the laboratory. A wide temperature range should be tested, especially at low temperatures since a resin may spend a great deal of time at lower temperatures if heating rates are slow. Further, the idea that the formula must correspond to some mechanistic expression is not important; of much more importance is that the functional form can predict the behavior for any resin and any set

\footnotetext{
${ }^{1}$ For certain situations, the density may also be important; however, the density is easy to determine. Note also the specification of material properties for example, the volume fraction of fibers may change but this is not a fundamental material property.

${ }^{2}$ The authors recognize that the thermal conductivity is difficult to calculate. However, the issue is determining the fiber orientations and the fiber volume fractions; not the thermal conductivity if those parameters are known.
} 
of reasonable conditions. In other words, robustness of the software demands the same (or, at a minimum, very similar) functional form for all resins, with the only changes being in the values of the parameters. Experimentally determining the parameters for a particular resin must be straightforward for implementation in the field.

Another important parameter, which is related to material properties, is the spatial distribution of temperatures. The thermal conductivity and the heat capacity will have a large effect on the temperature at a given point; also playing an important role is the heat released, both in a cumulative and in an instantaneous sense. Differential scanning calorimetry (DSC) is the most common method used to characterize the relationship between heat release and fractional conversion, as well as fractional conversion and time-temperature. In this method, heat evolved or required for a given timetemperature profile is monitored, and the fractional conversion $(\alpha)$ at any time $t$ can be determined by:

$$
\alpha=\frac{1}{H_{\mathrm{t}}} \int_{0}^{t}\left(\frac{\mathrm{d} H(t)}{\mathrm{d} t}\right) \mathrm{d} t
$$

$H$ is the enthalpy at any time $t$ and the signal from the DSC, properly normalized, is the quantity inside the brackets, i.e., $\mathrm{d} H / \mathrm{d} t$. The rate of change of fractional conversion is used in most model fits, where the rate of change of fractional conversion is given by:

$$
\frac{\mathrm{d} \alpha}{\mathrm{d} t}=\frac{1}{H_{\mathrm{t}}} \frac{\mathrm{d} H}{\mathrm{~d} t}
$$

$H_{\mathrm{t}}$ is the total enthalpy for the reaction, i.e., the integral in the above equation under the conditions of complete reaction. This expression implicitly ignores any prereaction of the resin, i.e., at time $t=0$, the fractional conversion is assumed to be zero. Practically, $H_{\mathrm{t}}$ is determined by measuring the heat of reaction at a constant heating rate, typically $20 \mathrm{~K} / \mathrm{min}$, being sure to begin and end at temperatures beyond the range where the reaction occurs. For an isothermal experiment, i.e., where the temperature is held constant and the heat released or gained is measured as a function of time, the enthalpy at infinite time $\left(H_{\infty}\right)$ may or may not be equivalent to the enthalpy measured during a constant heating rate experiment. If $H_{\infty}<H_{\mathrm{t}}$, then $\alpha_{\mathrm{r}}$, the relative conversion, can be calculated according to Equation (2) with $H_{\infty}$ replacing $H_{\mathrm{t}}$. With this convention, $\alpha_{\mathrm{r}}$ is equal to one by definition at infinite time for any temperature.

As mentioned before, viscosity is a very important material property during the early stages of reaction. This parameter is particularly important in predicting the existence or absence of voids. Oscillatory rheology is used to characterize the time-temperature relationship. Using this method coupled with DSC experiments, the relationship between viscosity and conversion can be characterized. In rheological experiments, a sample is sinusoidally strained at a fixed frequency and the sinusoidally varying stress is measured. Both the magnitude of the stress and the phase lag of the stress relative to that of the strain are measured; if the sample deformation is in the linear region, then the result will not depend on the strain magnitude. Oscillatory measurements have almost exclusively been performed with torsion instruments. The in-phase part of the stress is proportional to the storage modulus $\left(G^{\prime}\right)$ or alternatively the imaginary part of the viscosity $\left(\eta^{\prime \prime}\right)$; while the 
out-of-phase part is proportional to the loss modulus $\left(G^{\prime \prime}\right)$ or alternatively the dynamic viscosity $\left(\eta^{\prime}\right)$. The two are related by the following equation:

$$
\frac{G^{*}}{i \omega}=\frac{G^{\prime}+i G^{\prime \prime}}{i \omega}=\eta^{*}=\eta^{\prime}-i \eta^{\prime \prime}
$$

The important viscosity for composite manufacture cannot be measured in an oscillatory experiment, since the shear viscosity $(\eta(\gamma))$ is the relevant viscosity for compaction and consolidation. The steady-shear viscosity cannot be measured at reaction temperatures because of the time required to collect data relative to the cure rate, and hence most experimenters have used the Cox-Merz empirical relationship, which relates the steady-shear and complex viscosity according to the following equation:

$$
\eta^{*}(\omega)=\eta(\dot{\gamma}) \quad \text { at } \omega=\dot{\gamma}
$$

The utility of the Cox-Merz rule is at relatively large shear rates; in the limit of zeroshear or zero-frequency, the dynamic viscosity $\left(\eta^{\prime}(\omega)\right)$ and the steady-shear viscosity $\eta(\gamma)$ are the same. Further, the Cox-Merz empirical relationship was developed for systems without large elastic (in-phase) components, which is definitely not the case in reacting thermosets. Nonetheless, the measurement of the complex viscosity is by far the most common method to determine the viscosity for use in models that predict composite behavior during curing.

The focus of this study is not on the measurements of viscosity, rather our concern is the use of the storage modulus to measure the reaction rate. The reason this approach has merit is that the storage modulus is directly related to the stiffness of the composite, and the stiffness is one of the properties vitally important for any composite. The storage modulus versus time measured at isothermal temperatures has a shape qualitatively identical to that of the energy released versus time. Hence, using the storage modulus, one can define an extent of reaction [1,2] as shown in Equation (5). Note that the assumption in this model is that $G^{\prime}(0)=0$, which is experimentally accurate for almost all systems.

$$
\begin{aligned}
\alpha & =\frac{1}{\text { Normalization }} \int_{0}^{t}\left(\frac{\mathrm{d} G^{\prime}(t)}{\mathrm{d} t}\right) \mathrm{d} t \\
\frac{\mathrm{d} \alpha}{\mathrm{d} t} & =\frac{1}{\text { Normalization }} \frac{\mathrm{d}\left(G^{\prime}(t)\right)}{\mathrm{d} t}
\end{aligned}
$$

One might question why the conversion was defined without taking the logarithm of the storage modulus first. One obvious issue is that $G^{\prime}(0)$ can no longer be assumed to be zero, and accurate determination of $G^{\prime}(0)$ becomes important or some value of $G^{\prime}(0)$ must be assumed. The second issue is that using logarithms reduces the sensitivity to changes at long times, i.e., near the plateau region, and this region is of most interest in predicting the final properties.

One substantial problem with Equation (5) is that there is no counterpart in oscillatory rheological experiments for $H_{\mathrm{t}}$, i.e., a normalization constant invariant to the thermal history. The logical normalization constant is $G_{\max }^{\prime}$, i.e., the storage modulus as the reaction reaches completion, which is apparent as a plateau in the storage modulus versus time data. $G_{\max }^{\prime}$ definitely does depend on the curing temperature, as will be seen later. In isothermal cure experiments, the determination of $G_{\max }^{\prime}$ is straightforward; the issues 
surrounding nonisothermal cure conditions will be discussed more completely in the 'Results and Discussion' section.

One practical problem with measuring $G^{\prime}$ in a curing system is the very large change in modulus as the material cures, which not only creates instrumental issues, but also can cause difficulties in maintaining sample-plate contact, especially since most resins shrink during curing. As will be described later, definite evidence of inconsistent sample-plate contact was found when smooth plates were used to measure the storage modulus. The most common solution to improve sample-plate contact is to roughen the surface of the plates, or to attach sandpaper [3]. These modifications seem to have no effect on the measured rheological properties, although determining the correct properties under ideal conditions is not a trivial matter. However, we are not aware of a study on the effect of roughening plates with respect to measurements in a curing system, which is perhaps the most taxing experiment performed on a torsional rheometer. Simply roughening the plates, or using sandpaper, may not solve the problems inherent to measuring the rheological properties of curing systems. Our solution was to use grooved plates to better grip the sample; however, this modification changes the applied stress field substantially and could change the rheological properties observed. A related issue is that thicker samples can be required when grooved plates are used. Thicker samples could lead to higher internal sample temperatures, due to heating effects from the exothermic reaction.

A number of models have been developed to fit reactivity data acquired via DSC. A complete review of all the proposed models is beyond the scope of this article; however, some of the most popular models are discussed. In general, there are two types of models: an autocatalytic model and an $n$th order model. The former is used for systems where the rate shows a maximum as a function of percent conversion, while the latter is used for systems which have a maximum rate at the beginning of the reaction and the rate drops monotonically. The expression for the autocatalytic model is often termed the Kamal approach [4] and has the general form:

$$
\frac{\mathrm{d} \alpha_{\mathrm{r}}}{\mathrm{d} t}=\left(k_{1}+k_{2} \alpha_{\mathrm{r}}^{m}\right)\left(1-\alpha_{\mathrm{r}}\right)^{n}
$$

There have been a number of modifications to this model, the most common of which are $k_{1}=0$ and $\left(1-\alpha_{\mathrm{r}}\right)$ becoming $\left(\alpha_{\mathrm{r}(t=\infty)}-\alpha_{\mathrm{r}}\right)$ [5]. The latter change has the disadvantage of requiring absolute enthalpy determination. Although absolute enthalpy determination is not difficult in theory, the use of prepreg typically means that the resin has been prereacted to raise the viscosity and hence every raw material would have to be tested and unreacted resin would have to be supplied by the manufacturer, making this modification difficult to apply in practice. Hence, we chose to ignore this second modification, which seems to be justified because the quality of our fits was still quite good.

The $n$th order model has the form:

$$
\frac{\mathrm{d} \alpha_{\mathrm{r}}}{\mathrm{d} t}=k_{3}\left(1-\alpha_{\mathrm{r}}\right)^{n}
$$

For systems where the rate of reaction is determined by diffusion, $n=1$. Assuming the fits are satisfactory, the $k_{i}$ values in Equations (6) and (7) have been found to be well-described by an Arrhenius type of relationship, i.e., $k_{i}=A_{i} \mathrm{e}^{-E_{\mathrm{a}} / R T}$. To determine the parameters for these models, data are collected for a series of samples using different isothermal curing 
conditions. At each temperature the model is fit to the data, the constants are collected and then are fit to the appropriate expression.

From the point of view of an accurate description of the data, in some cases neither model provides an acceptable description of the experimental data over the entire range of conversions. One approach is to define two regions: one where the Kamal model is used and another where the diffusion model is used [6,7]. The so-called Springer-Loos model [7] is based on this approach; however, the form of the autocatalytic term is slightly different:

$$
\frac{\mathrm{d} \alpha}{\mathrm{d} t}=\left(k_{1}+k_{2} \alpha_{\mathrm{r}}\right)\left(1-\alpha_{\mathrm{r}}\right)\left(B-\alpha_{\mathrm{r}}\right)
$$

This type of expression does not have a relative maximum at intermediate cure states, unlike the normal Kamal expression.

An entirely different approach is to use an empirical expression, rather than one grounded in some fundamental kinetic principles, to fit reaction rate data. A polynomial is probably the simplest empirical expression to use. Given enough terms, a polynomial will give an almost exact description of the data at a given isothermal cure temperature. Equation (8) suggests a cubic polynomial as shown in Equation (9); a cubic polynomial agrees with the common convention that $m+n=2$ in the Kamal model.

$$
\frac{\mathrm{d} \alpha_{\mathrm{r}}}{\mathrm{d} t}=k_{1} \alpha_{\mathrm{r}}^{3}-k_{2} \alpha_{\mathrm{r}}^{2}+k_{3} \alpha_{\mathrm{r}}+k_{4}
$$

If no relationship exists between the coefficients at different temperatures, then this approach will be useless as a predictive tool. In other words, will an Arrhenius-type relationship describe the temperature dependence of the $k_{i} \mathrm{~s}$ in Equation (9)? In a recently published article [8], the answer was a definitive yes. This question is further explored in this present study.

The purpose of this study is to examine the calorimetric and rheological behavior of three different commercial epoxy resins used extensively as the matrix in commercial prepregs. The first is 3501-6; this type of resin and its prepreg, AS4/3501-6, have been studied extensively over 20 years, and our results are compared to that of previous studies. The second is Bryte BT250E-1 and the third is Newport NC301.

\section{EXPERIMENTAL}

\section{Materials}

All materials were obtained directly from the manufacturers: Hexcel for 3501-6, Bryte Industries for the Bryte BT250E-1, and Newport Adhesives and Composites for Newport NC301. We appreciate enormously the contributions of each company to this research. The resins were prepared in such a way that they were identical to the resin immediately before impregnation with fiber, i.e., immediately prior to the manufacture of the prepreg. Presumably the materials were prereacted to increase the viscosity for better impregnation behavior; we will provide enthalpic evidence that this was carried out for the 3501-6. For the other two resins, the enthalpy of the totally unreacted resin is not known, so we cannot say for sure what the cure state was of the resin that we tested. 


\section{DSC Experiments}

Differential scanning calorimetry experiments were carried out on a TA Instruments Q-1000. The same heating rate as for the rheology experiments, $27.8^{\circ} \mathrm{C} / \mathrm{min}$, was used prior to reaching the isothermal cure temperature to facilitate direct comparison between the data sets. After the samples had reached the maximum level of cure at that temperature, the sample was cooled to slightly above room temperature and scanned at $1{ }^{\circ} \mathrm{C} / \mathrm{min}$ to determine the glass transition temperature. For some samples, there was definitely evidence of further curing at temperatures higher than the measured glass transition temperature. At least two samples were run for each temperature to assure reproducibility; the samples were considered identical when the variation in any individual rate at a particular relative conversion was no more than roughly $10 \%$ and the qualitative shape of the curves were identical. Roughly $20 \%$ of the runs were judged to be unacceptable by this criterion, presumably due to poor sample-pan contact. Fits were performed by minimizing the square of the error between all data sets judged to be the same within experimental error. Resins were also scanned at various rates and $H_{\mathrm{t}}$ was determined by integrating the area under the curve with a linear baseline. Indium was used as both a temperature standard and an enthalpy standard.

\section{Oscillatory Shear Rheometry - Normal (Smooth) Parallel Plates}

Rheology experiments were carried out on a Rheometrics RDAIII using $12.7 \mathrm{~mm}$ diameter parallel plates. Enough material for a thickness of $\approx 1.0 \mathrm{~mm}$ was loaded into the machine, and the temperature was raised at $27.8^{\circ} \mathrm{C} / \mathrm{min}$ until the desired temperature was reached, and the material was held at that temperature until the storage modulus reached its plateau value. The rate of temperature increase was experimentally determined as the maximum rate where the sample followed the commanded temperature rise. The maximum rate was desired to minimize any reaction that might occur prior to reaching the desired cure temperature. The test was conducted at a frequency of $1 \mathrm{~Hz}$ and the autostrain feature was used; the initial strain was set to 0.5 . At this point, the sample was cooled to room temperature, and then a temperature scan at $1{ }^{\circ} \mathrm{C} / \mathrm{min}$ was carried out to determine the glass transition temperature $\left(T_{\mathrm{g}}\right)$. The $T_{\mathrm{g}}$ was determined according to the point at which the storage modulus $\left(G^{\prime}\right)$ began to drop-off due to softening of the material. Sample heating due to the exothermic nature of the reaction was minimal because of the rather thin samples used. The viscosity results (i.e., the loss modulus) are not discussed in this study because the range of temperatures and times investigated meant that the viscosity rose to very high levels very quickly and hence the data were not very interesting from a manufacturing or modeling perspective.

\section{Oscillatory Shear Rheometry - Grooved Plates}

Oscillatory shear rheometry experiments were also performed with an Alpha Technologies APA rheometer using $41.3 \mathrm{~mm}$ diameter parallel plates using the same cure temperatures as with the RDAIII. The APA is a much more robust rheometer than the research grade RDAIII; however, this rheometer does not have the extreme sensitivity of the RDAIII. The APA has no autostrain feature, which meant a strain of $0.5 \%$ was 


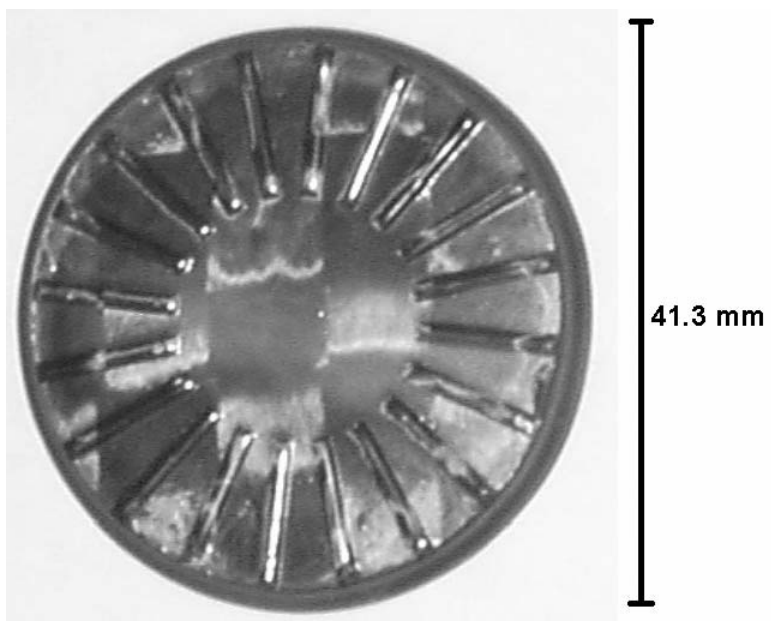

Figure 1. APA sample; a checkered carbon fiber composite was used for contrast. Sample diameter is $41.3 \mathrm{~mm}$ as indicated. There are a total of 20 ridges on each side of the sample, each with a height of $0.25 \mathrm{~mm}$. The ridges are equally spaced and arranged in a radial fashion. The thickness (width) of the protruding ridges is a constant $1.5 \mathrm{~mm}$, and the ridges are $15 \mathrm{~mm}$ long.

used throughout the experiment. The constant strain caused no noticeable problems with data quality since the APA is capable of measuring a much wider range of forces. The APA's design requires a constant sample thickness because the gap size cannot be varied; so the sample amount was carefully measured and maintained for a given gap setting. Changes in the sample amount did change the value of $G_{\max }^{\prime}$ measured.

The APA's plates are designed with grooves to prevent resin slippage; a cured sample is shown in Figure 1. The normal sample thickness is $2.5 \mathrm{~mm}$; however, because of possible exotherm artifacts, for one set of measurements the instrument was modified so that the sample thickness was $1 \mathrm{~mm}$ instead of $2.5 \mathrm{~mm}$, as noted in the text. Note that the numbers given represent the minimum thickness, i.e., the thickness corresponding to the valleys between the grooves, or the thickness in the constant thickness center portion of the sample. The actual thickness at a position at a radial distance corresponding to the location of the grooves was between 1 and $1.5 \mathrm{~mm}$, or 2.5 and $3 \mathrm{~mm}$, respectively, depending on the alignment of the grooves. In calculations of moduli, the minimum thickness was used.

\section{Oscillatory Compression Rheometry}

We attempted to make the same measurements using compressive oscillatory rheometry experiments with a Rheometrics RSA-II. The plates used ranged in size from 25 to $4 \mathrm{~mm}$. Even with the smallest plates, machine compliance was too large at high degrees of cure, resulting in artificially low values of the modulus. Hence, the results are not reported in this study. There is one report of using oscillating plates in compression to make measurements on curing systems; the machine design was much different which resulted in a much higher stiffness instrument [9]. However, at lower fractional conversions where machine compliance was not an issue $(\alpha<0.25)$, the rate of property development was similar to the rate measured with the shear smooth and grooved geometries; in fact the 
data quality in compression was much higher than that in shear. It has been shown previously that when the sample radius is large in comparison to the sample thickness, the flow field is dominated by shear deformation [10], and hence the cure rate measured in compression was expected to be identical to those values measured in torsion.

\section{RESULTS AND DISCUSSION}

\section{Results of Calorimetric Studies}

Three studies have measured heat release as a function of isothermal curing temperature in a DSC for 3501-6 [7,11,12], and two other studies measured these quantities in AS4/ 3501-6 prepreg and adjusted the measured value by the measured weight fraction of fibers [6,12]. The values of $H_{\mathrm{t}}$ at a $20^{\circ} \mathrm{C} / \mathrm{min}$ heating rate for the pure resin differ slightly: $473.6 \pm 5.4 \mathrm{~J} / \mathrm{g}$ by Lee et al. [7], $502 \pm 21 \mathrm{~J} / \mathrm{g}$ by Hou and Bai [10], and most recently, $508 \pm 19 \mathrm{~J} / \mathrm{g}$ by Chern et al. [12] with errors representing two standard deviations. No specific explanation was offered by Chern et al. as to why the value presented by Lee et al. was different. Chern et al. measured the enthalpy of the resin in AS4 graphite fiber/3501-6 prepreg and found it to be 433.7 or $422.7 \mathrm{~J} / \mathrm{g}$ depending on baseline subtraction [6], which agreed with the value reported by White and $\mathrm{Kim}, 435 \mathrm{~J} / \mathrm{g}$ [13]. Chern et al. attributed the lower value versus the value for the pure resin to the changes in the cure mechanism due to the presence of the fiber; prereacting the resin was ruled out based solely on statements from the manufacturer. Regarding the heat of reaction for prepreg, the total enthalpy depended on the heating rate of the DSC scan.

Figure 2 shows that in this study the total enthalpy did depend, at least weakly, on the scan rate for 3501-6; for the other two resins, there was no dependence of the total enthalpy on the scan rate in dynamic measurements. In the previous study on 3501-6 prepreg where the enthalpy depended on the scan rate [6], the enthalpy decreased

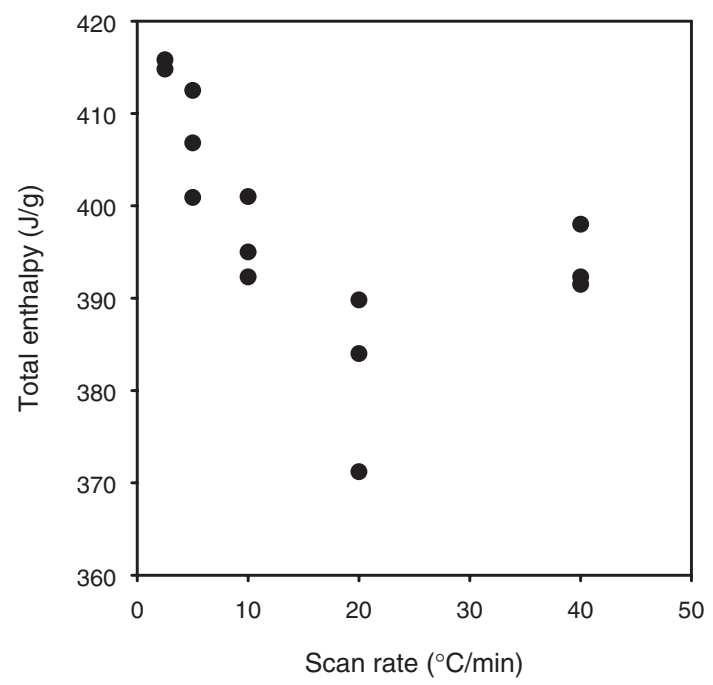

Figure 2. Heat of reaction for 3501-6 during dynamic scans as a function of scan rate. 
monotonically with an increasing scan rate rather than reaching what appears to be a plateau as seen in this study. Other studies that investigated epoxy resins found monotonic decreases as well; however, the highest scan rate investigated in these studies was $20^{\circ} \mathrm{C} / \mathrm{min}$ $[14,15]$. Presumably, the reason for the dependence of the enthalpy on the scan rate is the time lag associated with faster scan rates, which in turn causes faster hardening of the resin and prevents full reaction. Isothermal scans also show that fast hardening can occur and prevent full reaction. Within experimental error, there was no difference in the glass transition temperatures of the materials cured at various scan rates; this result differs from what has been found previously [14]. The value of the enthalpy at a scan rate of $20^{\circ} \mathrm{C} / \mathrm{min}$ is $382.5 \pm 20 \mathrm{~J} / \mathrm{g}$, more than $20 \%$ less than the enthalpy of the unreacted material, i.e., roughly $500 \mathrm{~J} / \mathrm{g}$. Since fibers were not present in the material used in this study, the only explanation is that the prereaction step for this resin was more significant than that reported previously [6].

The enthalpy as a function of isothermal curing temperature is shown in Figure 3. Regarding the 3501-6, $H_{\mathrm{t}}$ (represented by the dotted line on the graph) is, in all the cases,
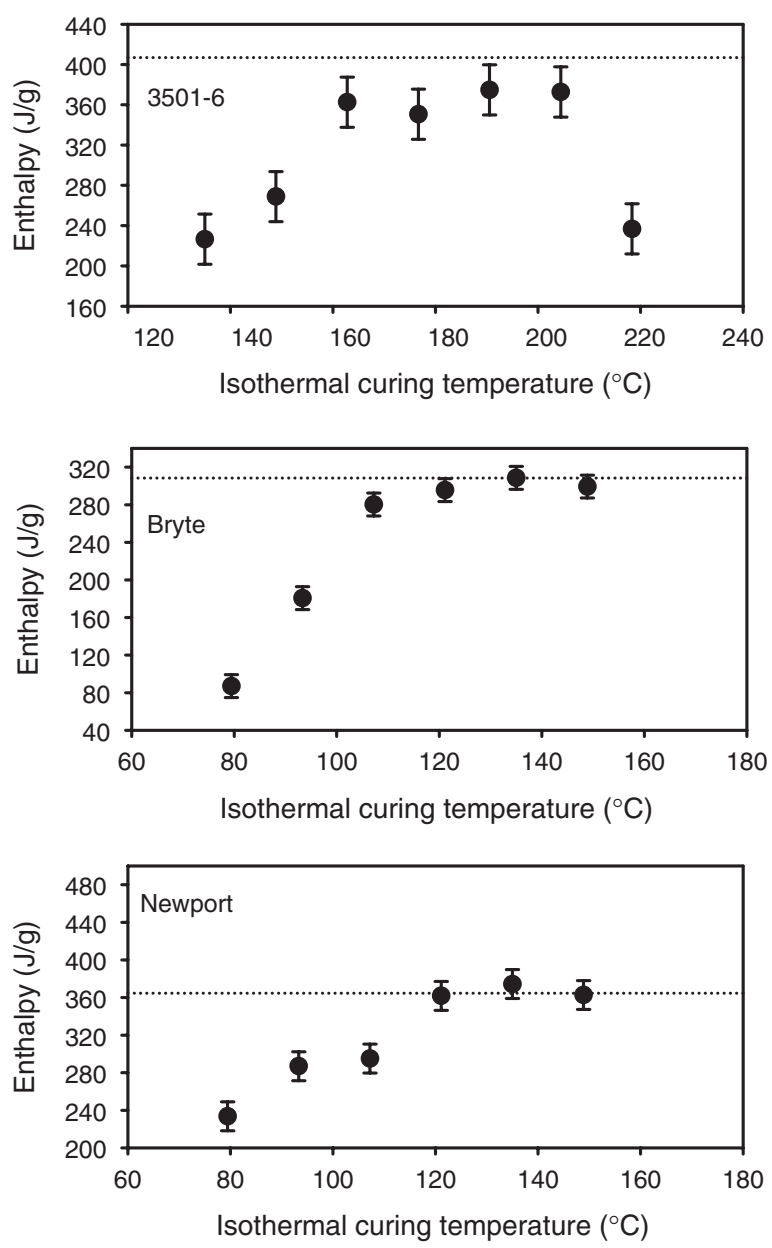

Figure 3. Heat of reaction at different isothermal curing temperatures. 
higher than the enthalpy measured in isothermal experiments, while for the other two resins, the total enthalpy in the isothermal scans was, at least in some cases, identical to that in dynamic scans. The reason for this difference is shown explicitly by Figures 9-11; the rate of reaction near the beginning of the reaction is very large for the 3501-6, while the opposite is true for the Newport and Bryte. In other words, some reaction likely occurs during the heat-up at $27.8^{\circ} \mathrm{C} / \mathrm{min}$ for the $3501-6$, which is not accounted for in the measurements (i.e., integration began when the isothermal temperature was reached). Only the 3501-6 shows a lower enthalpy at the highest isothermal temperature tested, indicating that at too high a temperature, the reaction will not fully proceed to completion. The other two resins do not show a reduced enthalpy at higher curing temperatures. However, as will be seen in the measurement of the storage modulus, the stiffness is significantly degraded if the curing temperature is too high for all the three resins.

Very surprisingly, the $T_{\mathrm{g}} \mathrm{s}$ shown in Figure 4 show very different qualitative behaviors versus the enthalpy. In general, the lower the enthalpy, the lower the $T_{\mathrm{g}}$, but this statement was not found to be true in all instances. The most glaring example is found for the 3501-6 sample cured at $218^{\circ} \mathrm{C}$; the conversion is far below $100 \%$, yet the $T_{\mathrm{g}}$ shows no indication
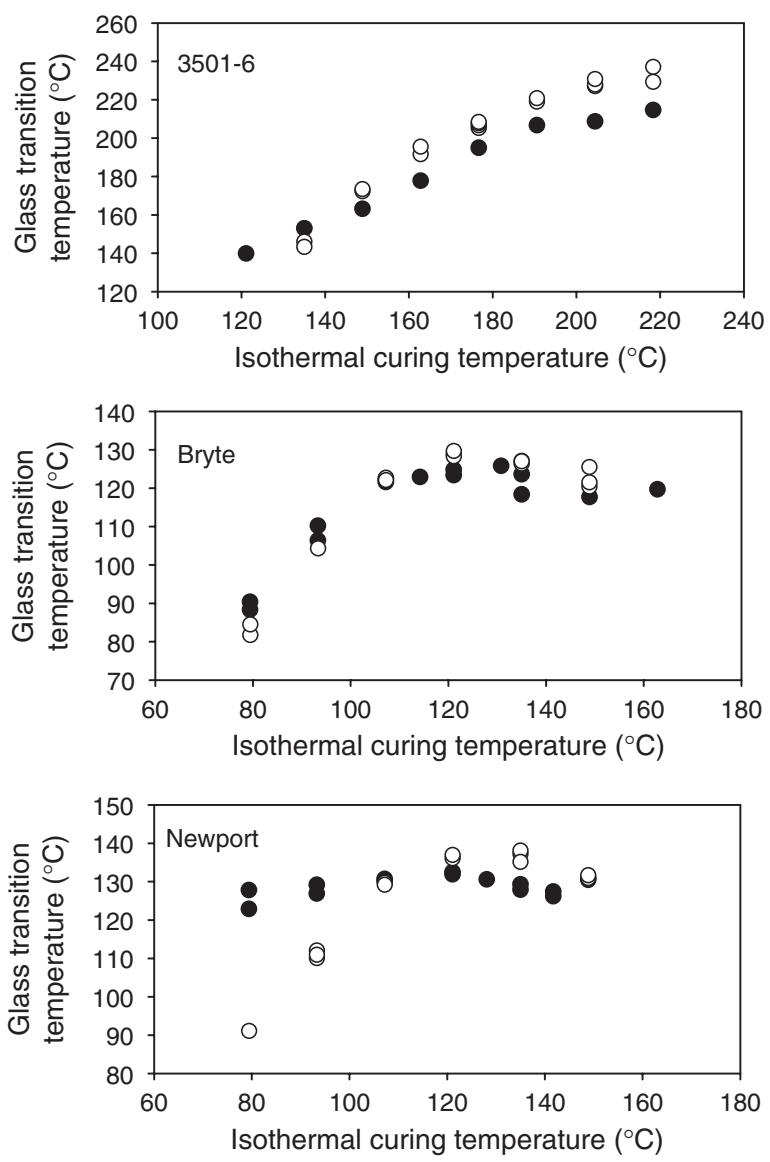

Figure 4. Glass transition temperature measured at different isothermal curing temperatures. Filled symbols are values from rheometry, while open symbols are values from DSC. 
of a reduced conversion. The Newport at $107^{\circ} \mathrm{C}$ also shows a $T_{\mathrm{g}}$ suggesting full cure, while the enthalpy shows a degree of cure more than $20 \%$ lower than the maximum. The maximum value of $G^{\prime}$ was not correlated with the $T_{\mathrm{g}}$ or the total enthalpy released upon cure over the entire range of cure temperatures. This observation contradicts the observation made by Malkin et al. for phenolic resins [2], i.e., the $T_{\mathrm{g}}$ was directly related to the degree of cure.

\section{Comparison between Smooth and Grooved Plates}

Figure 5 shows the maximum observed storage moduli $\left(G_{\max }^{\prime}\right)$ versus cure temperature for each resin and rheometer type. The run-to-run variation in $G_{\max }^{\prime}$ was quite large with the smooth plates, which the authors attribute to inconsistent sample-plate contact. The statistical variation of $G_{\max }^{\prime}$ was much lower for the grooved plates relative to the smooth
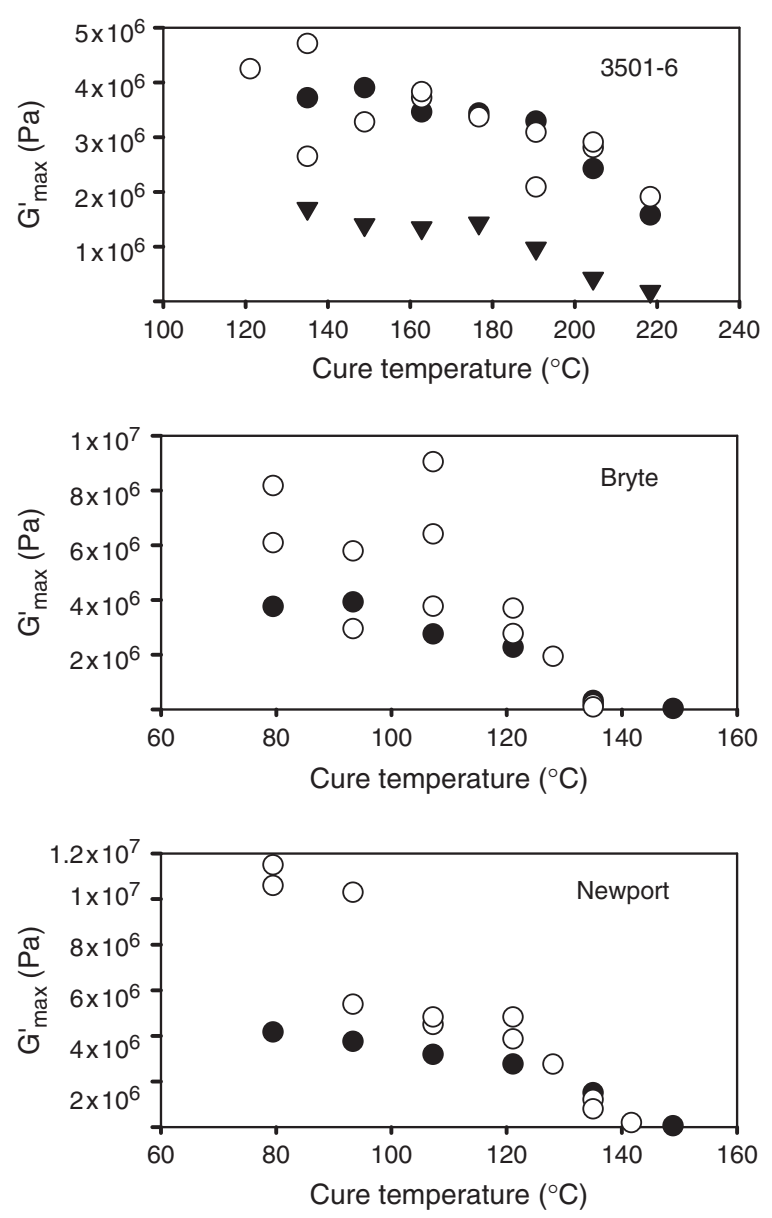

Figure 5. Maximum storage modulus observed vs cure temperature of the three different resins. Filled circles represent grooved plates with a nominal $2.5 \mathrm{~mm}$ sample thickness, open circles represent smooth plates, and filled triangles represent grooved plates with a nominal $1 \mathrm{~mm}$ sample thickness. 
plates, and the measured values of the storage moduli using the grooved plates and the thick sample were statistically within the large error of the values measured using smooth plates.

However, the values of the storage moduli were different clearly for the samples with different thicknesses measured with the grooved plates. Different sample thicknesses cannot result in different values of the measured storage moduli, since measurements were made in the linear viscoelastic region. The difference is believed to be related to how the sample deforms in the grooved plates. It is interesting to note that the difference between the moduli was approximately equal to the ratio of the two thicknesses. In other words, the measured torques for the two materials were approximately the same, instead of the factor of 2.5 difference that should have been seen if true parallel plate geometry were applicable. The factor of 2.5 difference was also seen in the modulus versus temperature curve used to determine the $T_{\mathrm{g}}$ after isothermal curing. The logical explanation for this observation is that what is actually being measured is the deflection of the ridges, and this deflection should be approximately independent of overall thickness since the ridge dimensions do not depend on overall thickness. Of course, if the material is too thin, then this approximation will break down, since at some point the ridges will not be the only part of the resin that deforms.

Although the values of $G_{\max }^{\prime}$ depend on sample thickness, the rate of cure does not. Figure 6(a) depicts the shear storage modulus $\left(G^{\prime}\right)$ versus time for $3501-6$ cured at $350^{\circ} \mathrm{F}$. Since the cure model predicts the rate of cure to the maximum conversion $(\alpha=1)$, the data must be normalized (i.e., the storage moduli converted to fractional conversions) for comparison between different rheometers. Figure 6(b) depicts the fractional conversion versus time generated from the data in Figure 6(a). As shown in Figure 6(b), the rates measured from different shear grooved sample thicknesses are statistically identical, and this result was found for nearly all cure temperatures. This result is particularly noteworthy because the maximum storage moduli measured from the different sample thicknesses were a factor of 2.5 times different; in other words, although the measured properties are different, they develop at the same rate. Note also that equivalent rates mean that the exotherm does not cause significant temperature gradients in the sample, at least for these resins. To better compare the rates for all temperatures and resins, the time to $80 \%$ cure $(\alpha=0.80)$ for each resin and temperature is presented in Figure 7 . These figures clearly show that the cure rate is independent of whether grooved or smooth plates are used. Using the time to $50 \%$ and $60 \%$ cure instead of $80 \%$ cure also showed that the rate did not depend on whether grooved or smooth plates were used.

\section{Comparison between Oscillatory Shear Rheometry and Differential Scanning Calorimetry}

Figure 8 shows a direct comparison between the extent of reaction measured calorimetrically, i.e., from the heat evolved, and mechanically, i.e., from the change in the storage modulus, for the 3501-6. Note that the rheological data presented from this point forward represents data from the smooth plates; as noted in the previous section, the rates of the two sample-plate types are identical. This figure represents qualitatively the behavior for all resins in that the mechanical extent of reaction lags behind that measured by heat release. The lag depends on the resin, and higher lags occur at lower temperatures. In other words, the modulus builds over a longer time scale than heat is released. 

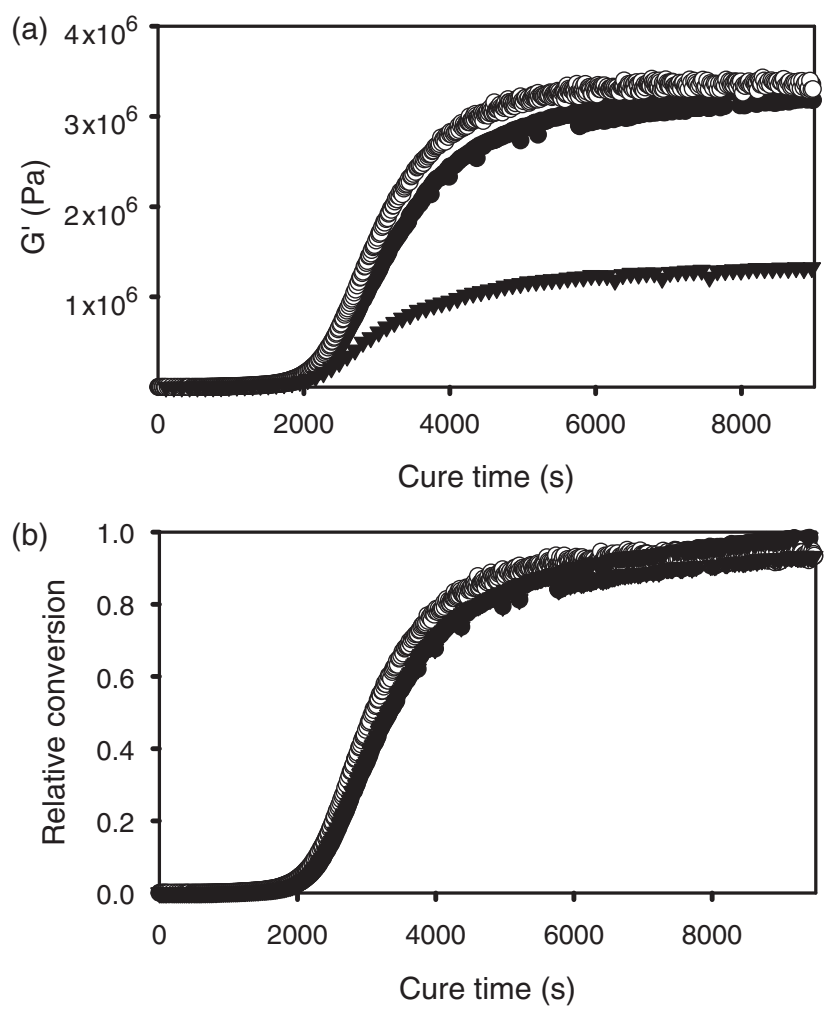

Figure 6. (a) Storage modulus vs cure time for 3501-6 cured at $350^{\circ} \mathrm{F}$ and (b) fractional conversion of 3501-6 cured at $350^{\circ} \mathrm{F}$ calculated from the data in part (a). Symbols are the same as those used in Figure 5.

The corollary to this statement, which is born out at some temperatures, is that the modulus continues to build slightly even when all measurable heat has been evolved. Based on these data, one cannot determine whether the lag is due to interchange reactions (which will result in no enthalpy change) or due to the heightened sensitivity of rheology experiments relative to calorimetric experiments at high degree of conversions. Another possibility is that the oscillatory strain in the rheology experiments allows the resin to react further than in a static DSC experiment. Further credence to this latter explanation is given by the difference in rheological $T_{\mathrm{g}} \mathrm{s}$ and DSC $T_{\mathrm{g}} \mathrm{s}$ for the Newport at $80-100^{\circ} \mathrm{C}$. The rheological $T_{\mathrm{g}} \mathrm{s}$ indicate that a much higher extent of reaction was reached versus the $T_{\mathrm{g}} \mathrm{S}$ measured via DSC. However, neither the 3501-6 nor the Bryte showed such a divergence of $T_{\mathrm{g}}$, yet the lag was definitely present.

Rate versus conversion results for the three resins according to the DSC spectra (black solid line) and rheological measurements (gray solid line) are shown in Figures 9-11 along with some model fits. The following comments are made on Figures 9-11:

1. Third-order polynomial model fits to the DSC data are not shown in Figures 9-11 because in some cases, fits were non-physical at high conversions. Specifically, there was an upturn of the fitted curve at high conversions. Hence, the third-order model was eliminated from the consideration for describing DSC data. 

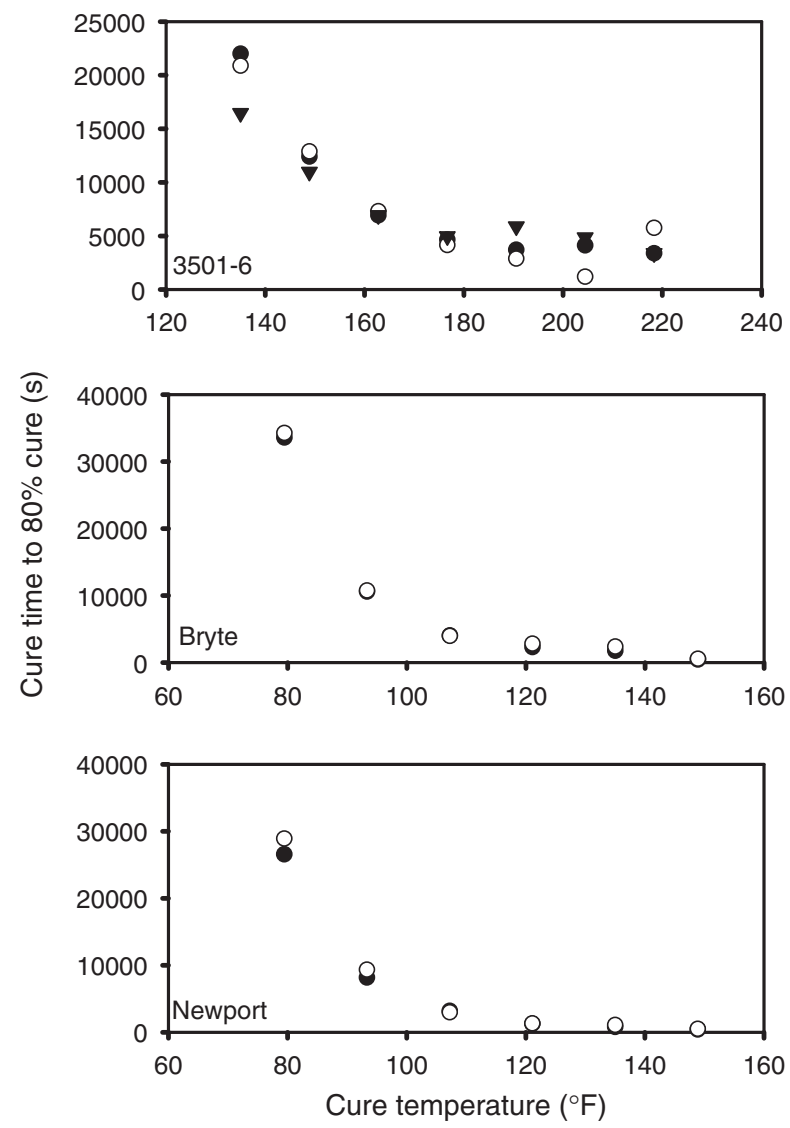

Figure 7. Cure time for $80 \%$ cure vs cure temperature for 3501-6, Bryte, and Newport. Symbols are the same as those used in Figure 5.

2. The Springer-Loos approach that consists of splitting the data into two parts, with the second part being described by the power-law model (Equation (7)) with $n=1$, gave satisfactory fits to data at some temperatures. However, at other temperatures the fit was not acceptable and hence this procedure was discarded. The veracity of these claims can be assessed in Figures $9-11$ by noting the linearity, or lack thereof, at high conversions.

3. For the Kamal autocatalytic model, when the data were fit with $k_{1}$ the fit was slightly better; however, $k_{1}$ varied in sign and hence fitting $k_{1}$ as a function of temperature was impossible. Therefore, $k_{1}$ was set to zero. The dependence of $m$ and $n$ on temperature was not easy to discern as shown by the representative data shown in Figure 12, a linear dependence was selected because of its simplicity. Linear dependencies, or slightly more complicated second-order polynomial dependencies, have been used previously to fit $m$ and $n[16]$.

The ability of the third-order polynomial, with Arrhenius dependencies of the $k_{i} \mathrm{~s}$, to predict the rate of modulus development as a function of conversion for the rheological data was mixed. For the rheological data, $k_{4}$ values did not vary in a regular manner with 

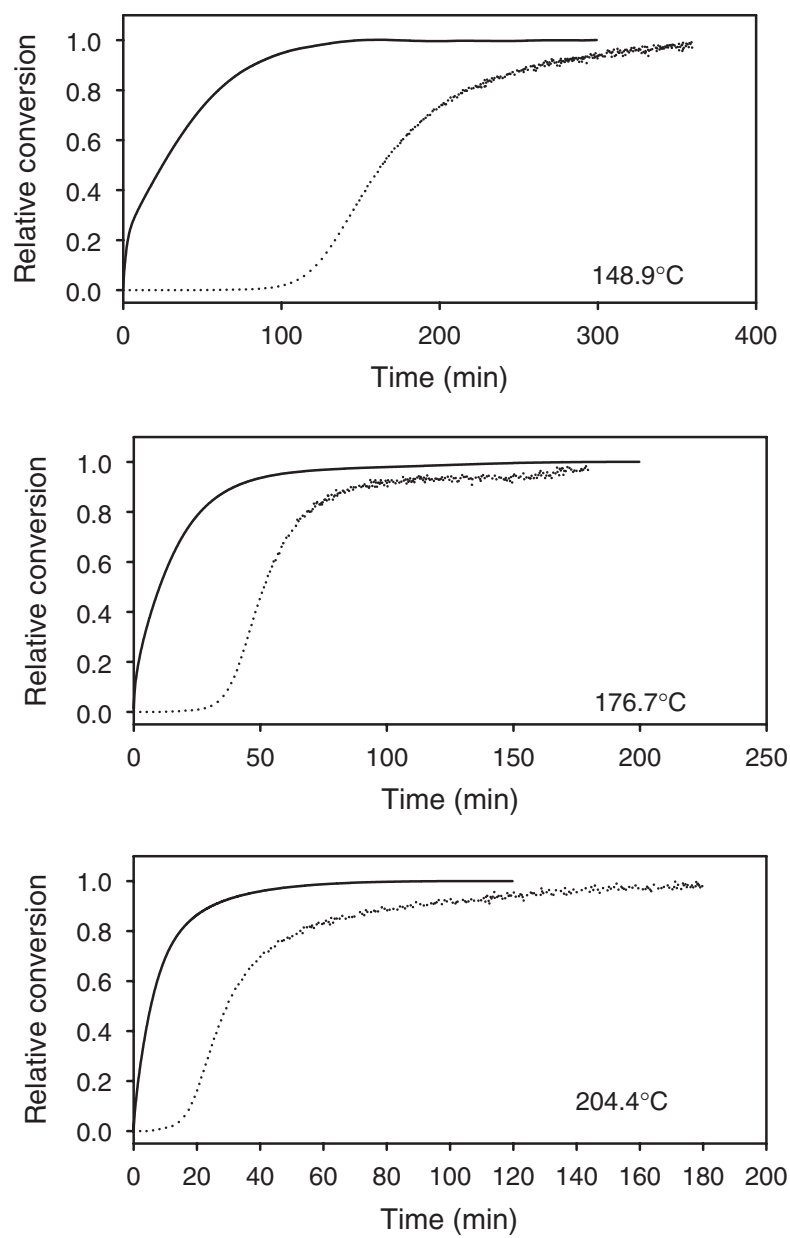

Figure 8. Percent conversion as a function of time for 3501-6. Solid lines are values from DSC, while dotted lines are values from rheometry.

temperature and tended to be quite small, and hence was set to zero in the predictions. In terms of the accuracy of the prediction, the third-order approximation worked relatively well for the 3501-6, as shown in Figure 9. For the other two resins, for some temperatures the prediction was quite close to the actual measured values, while for other temperatures the predictions were as much as factors of 2-3 different. For the third-order model to be useful, the dependence of the $k_{i} \mathrm{~s}$ with respect to temperature must be mathematically describable with a resin-independent functional form. As shown in Figure 13, an Arrhenius relationship is quite good except for the Bryte; the best-fit parameters are found in Table 1. The autocatalytic model on the average did not predict the actual rheological response any better than the third-order polynomial.

The activation energy can also be determined by the variation of the gel time with temperature assuming that the gel time represents an isoconversion point, i.e., the conversion is the same no matter the thermal history. However, it has been argued that the fractional conversion at the gel point has a temperature dependence [17]; this dependence may be contingent on how the gel point is measured. Many different methods have been 

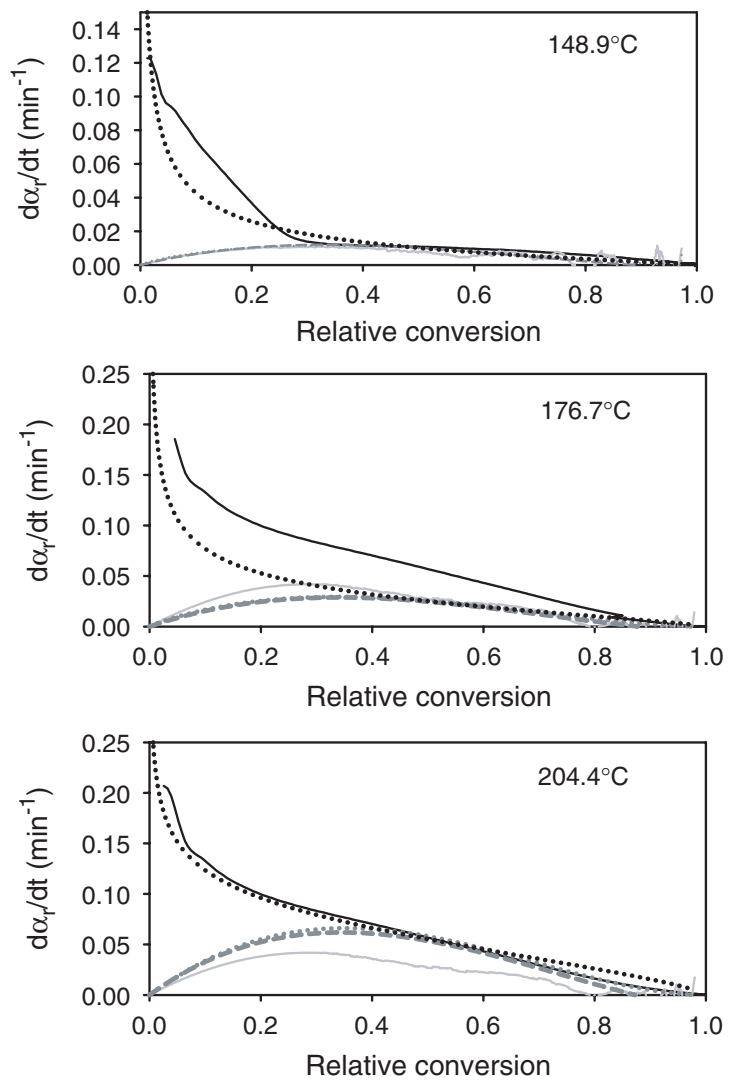

Figure 9. The rate of reaction vs conversion for three different isothermal curing temperatures as indicated on the graph for 3501-6. The solid black line represents experimental DSC data (Equation (2)) while the solid gray line represents experimental rheological data (Equation (5)) as indicated on the chart. The dotted black line represents model-predicted calorimetric data using the autocatalytic model (Equation (6)). The dotted gray line represents the model-predicted oscillatory rheometry data using the Kamal autocatalytic model, while the dashed gray line represents the third-order polynomial model (Equation (9)). The coefficients for the thirdorder polynomial model of the oscillatory rheometry data are given in Figure 13.

proposed and used to determine the gel point from oscillatory rheology data and a recent review article looked at the different methods critically [18]. For simplicity, the maximum in $\tan \delta$ was the method used to determine the gel point, and these times were plotted in the appropriate format, as shown in Figure 14. An Arrhenius expression did an excellent job of describing the data; of course a good fit does not prove that the gel time represents an isoconversion point. Comparison of activation energies from gel time measurements can be made with activation energies from enthalpic measurements, since ideally conversion is determined by the fraction of functional groups reacted for both. The gel time activation energy compares quite well with the activation energy determined using the Kamal expression for the 3501-6 (Table 2); but not as well for the other two resins $(\approx 15 \%$ difference). The activation energies for the third-order polynomial coefficients determined via rheology were also very different from the coefficients determined via the gel time or from the Kamal fit to the DSC data. This difference is possibly a result of using an empirical expression to describe the reaction rate, or could also be due to the fact that the 


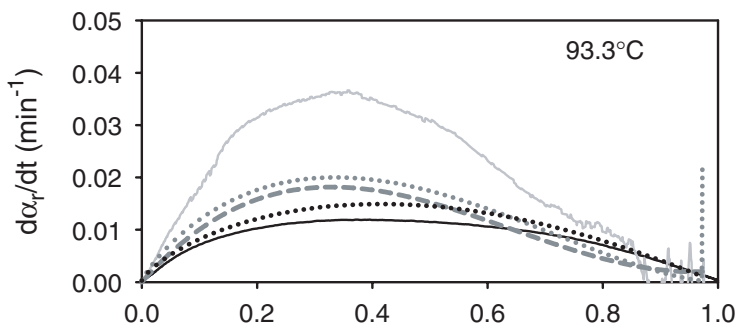

Relative conversion

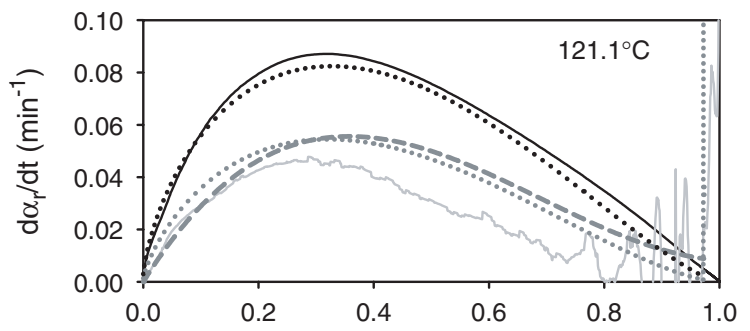

Relative conversion

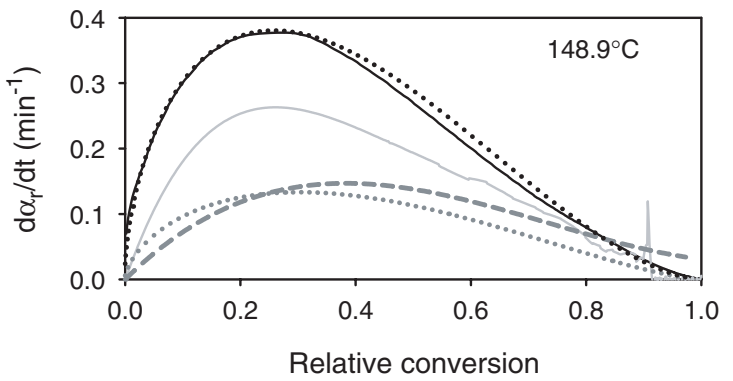

Figure 10. The rate of reaction vs conversion for three different isothermal curing temperatures as indicated on the graph for Bryte. The solid black line represents experimental DSC data (Equation (2)) while the solid gray line represents experimental rheological data (Equation (5)) as indicated on the chart. The dotted black line represents model-predicted calorimetric data using the autocatalytic model (Equation (6)). The dotted gray line represents the model-predicted oscillatory rheometry data using the Kamal autocatalytic model, while the dashed gray line represents the third-order polynomial model (Equation (9)). The coefficients for the thirdorder polynomial model of the oscillatory rheometry data are given in Figure 13.

DSC and rheological data are sensitive to very different things. Evidence supporting the latter is found in the fact that a Kamal fit to the rheological data also gave very different activation energies versus the gel point or the Kamal fit to the DSC data.

\section{Predictive Modeling for Storage Modulus}

Our desire is to simulate $G^{\prime}$, not the rate of change of $G^{\prime}$, for an arbitrary curing cycle. This section describes how one could use the results of this study to perform this task. To predict $G^{\prime}$, Equation (9) should be reformulated into a recursive relationship as:

$$
\alpha_{j+1}=\left(k_{1} \alpha_{j}^{3}-k_{2} \alpha_{j}^{2}+k_{3} \alpha_{j}+k_{4}\right) \Delta t+\alpha_{j}
$$



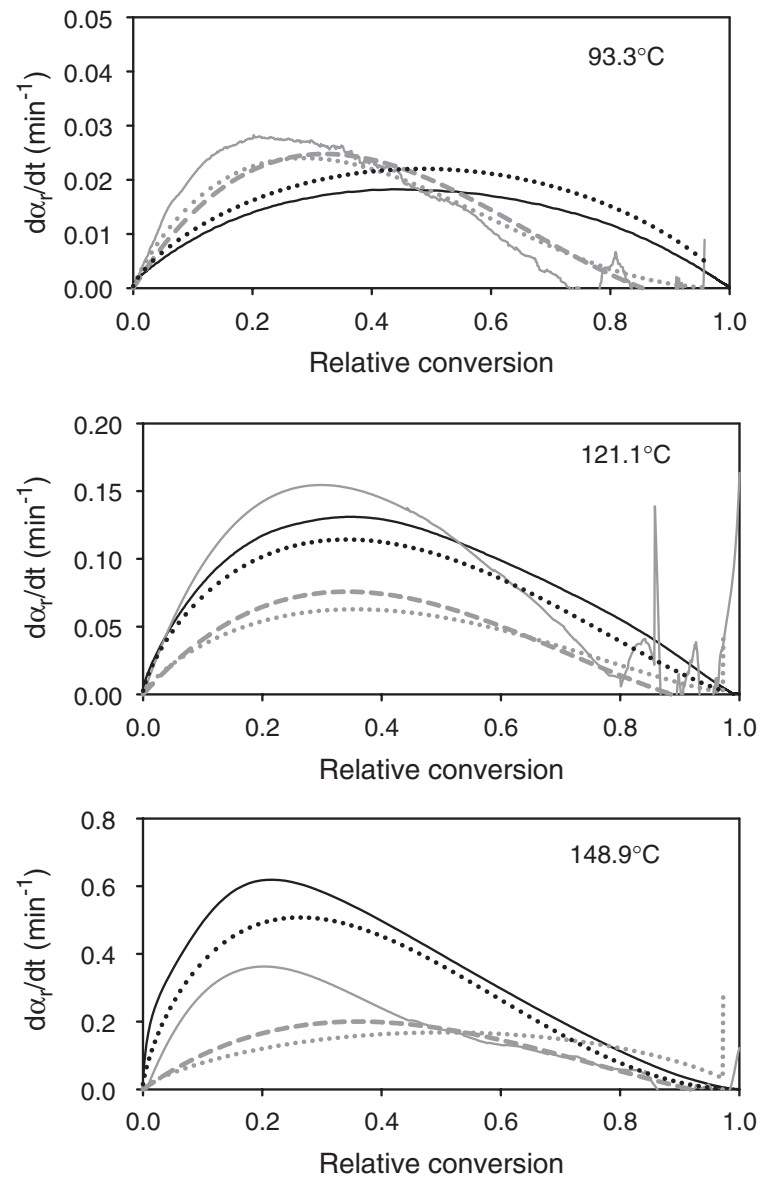

Figure 11. The rate of reaction vs conversion for three different isothermal curing temperatures as indicated on the graph for Newport. The solid black line represents experimental DSC data (Equation (2)) while the solid gray line represents experimental rheological data (Equation (5)) as indicated on the chart. The dotted black line represents model-predicted calorimetric data using the autocatalytic model (Equation (6)). The dotted gray line represents the model-predicted oscillatory rheometry data using the Kamal autocatalytic model, while the dashed gray line represents the third-order polynomial model (Equation (9)). The coefficients for the third-order polynomial model of the oscillatory rheometry data are given in Figure 13.

The constant term, i.e., $k_{4}$, cannot be zero in a recursive relationship, or else the conversion will always be zero. Our approach is to treat $k_{4}$ as a fitted parameter, i.e., fit $k_{4}$ while the data are being collected. Using $k_{4}$ in this manner has an added advantage of somewhat compensating for any modeling inconsistencies. One can envision two approaches using this strategy: choose a reasonable value for $k_{4}$ at the beginning, then choose an arbitrary conversion (5-20\% seems reasonable) and fit the data once to determine $k_{4}$; or, alternatively, fit $k_{4}$ all the way throughout the cure cycle. Clearly the latter is preferred; however, nonlinear least-squares fitting is a computationally intensive activity, and the approach will be driven practically by the speed of the computer used to control the process. One substantial disadvantage that exists when using $k_{4}$ as a fitted parameter is that the fractional conversion at infinite time will not be 1 ; a number of solutions exist to this problem. 

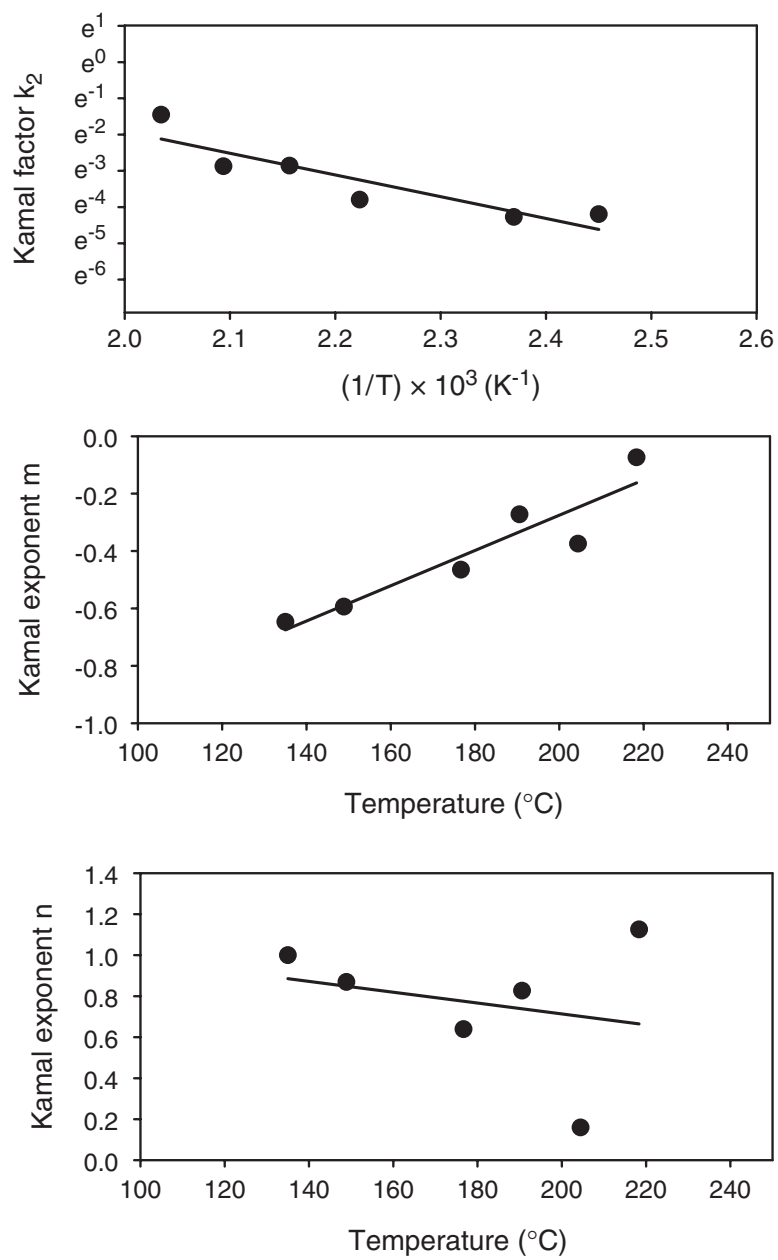

Figure 12. Fits for $k_{2}, m$, and $n$ to determine relevant Arrhenius, linear, and linear parameters respectively for 3501-6. Data for these plots were generated using isothermal cures in a DSC. As suggested by this plot, there was a great deal of scatter in the linear fits, while the scatter in the Arrhenius fit for $k_{2}$ was small for all resins.

The next step will be to transform the conversions calculated from Equation (10) into a storage modulus using Equation (5), which requires a value for the plateau storage modulus. $G_{\max }^{\prime}$ is essentially constant until some critical temperature is reached, at which time the modulus decreases linearly until an apparent modulus of zero is reached on this linear scale (in fact of course the modulus is not zero). The origin of this behavior results from the behavior of the $T_{\mathrm{g}}$ with cure temperature. As the cure temperature goes up, $T_{\mathrm{g}}$ reaches a plateau, as shown in Figure 4. Eventually, the plateau temperature is below the curing temperature of the resins. At curing temperatures above the $T_{\mathrm{g}}$ plateau temperature, the modulus at infinite time drops with further increases in temperature because the temperature becomes further away from the $T_{\mathrm{g}}$. The accuracy of this statement is shown by the behavior of the Bryte and Newport versus the 3501-6; the former show much clearer $T_{\mathrm{g}}$ plateaus and much stronger drops in the $G_{\max }^{\prime}$ with temperature. 

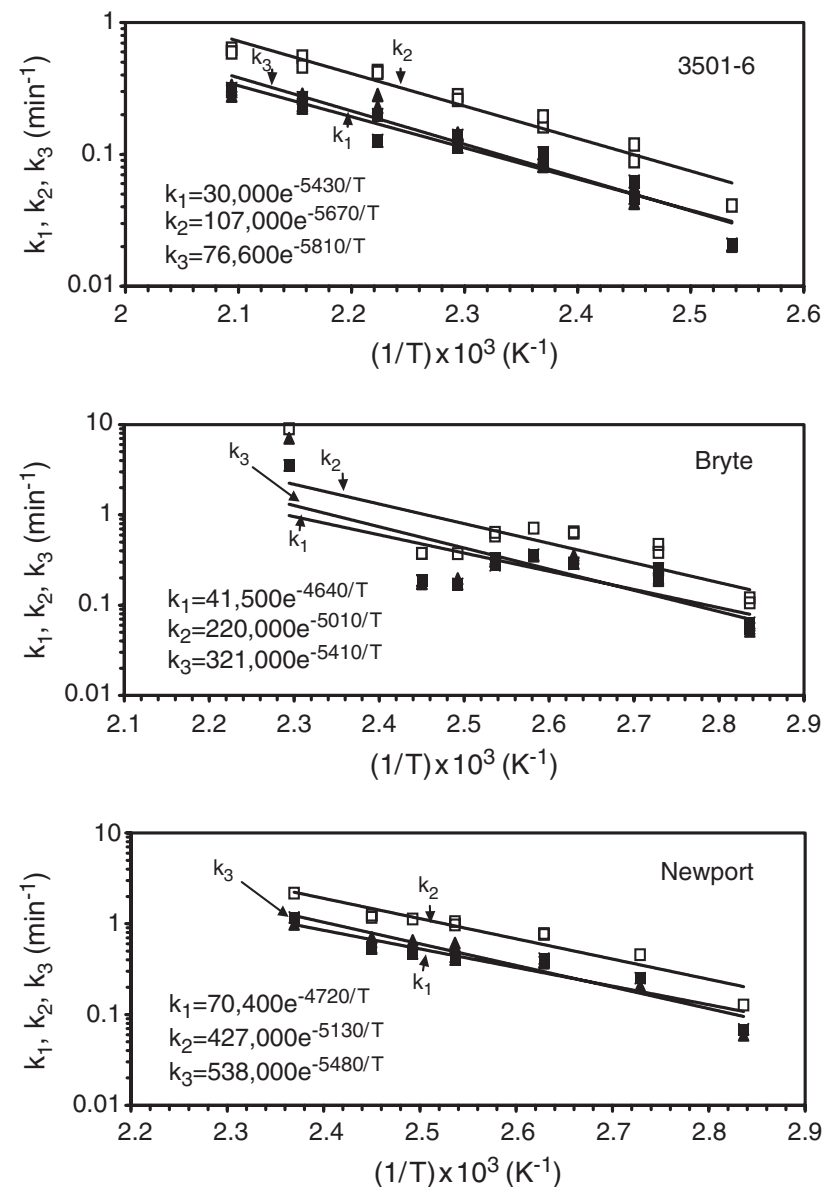

Figure 13. Fits for $k_{1}, k_{2}$, and $k_{3}$ to determine Arrhenius parameters for third-order model (Equation (9)). Data for these plots were generated using isothermal cures in an oscillatory rheometer.

Table 1. Parameters for third-order polynomial cure model.

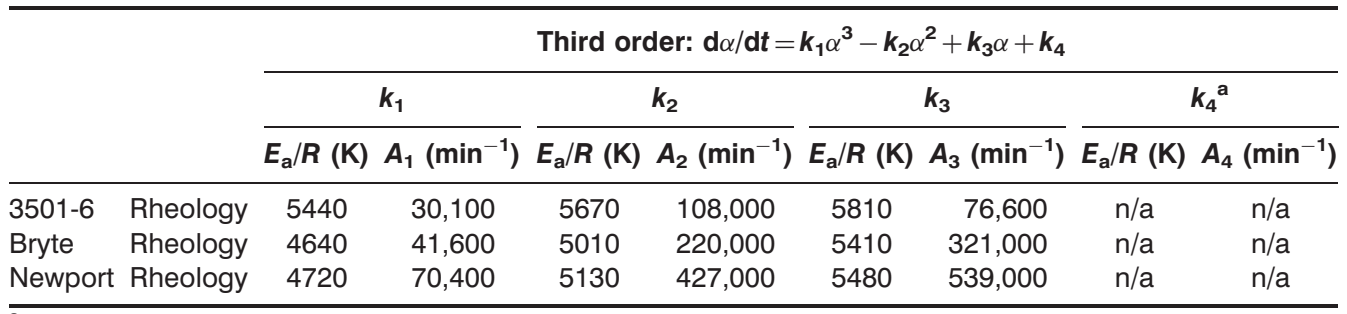

${ }^{a} k_{4}$ was very small and changed sign without any discernible pattern, and hence was set to zero to determine the best-fit $k_{1}-k_{3}$.

The desire of the authors is to have Equation (10) represent the mechanical fractional curing, and, on this basis, $G_{\max }^{\prime}$ should be set at the value corresponding to the value at the instantaneous curing temperature, since temperature determines $G_{\max }^{\prime}$. The implicit assumption is that mechanical fractional conversion is absolute no matter the thermal 

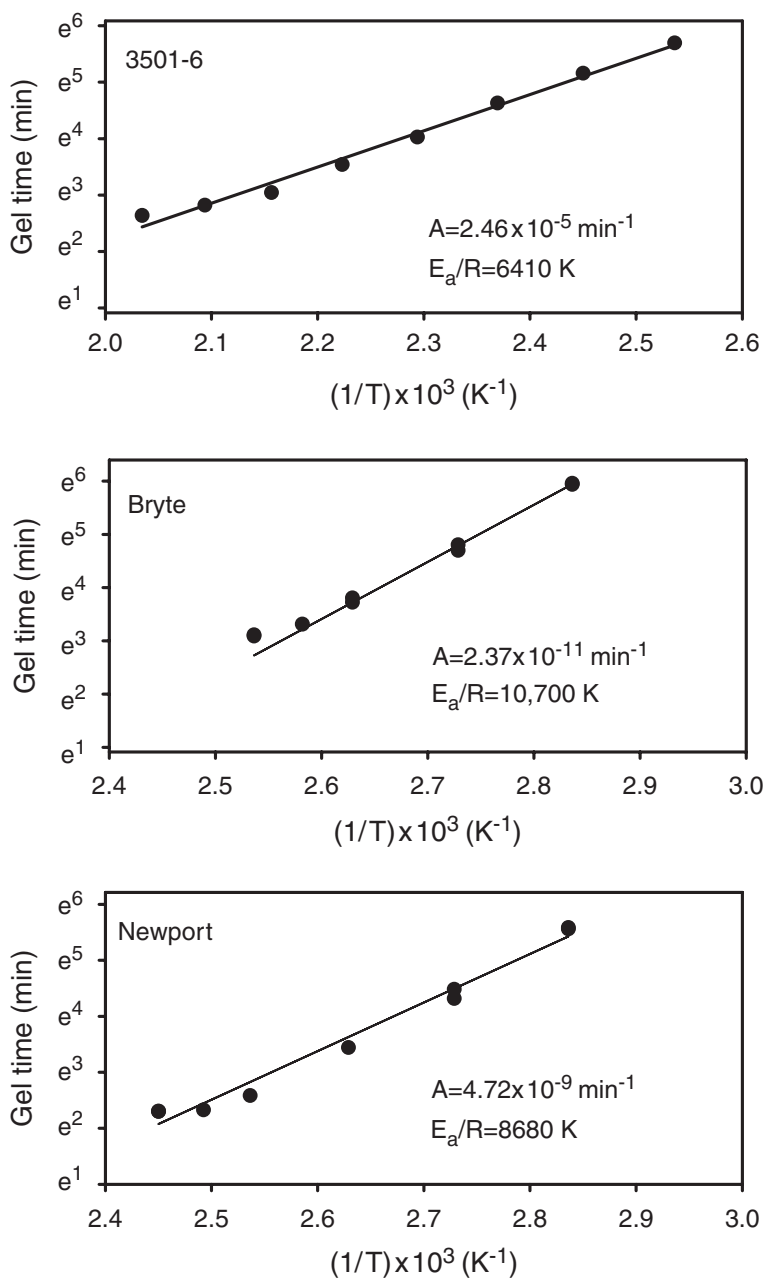

Figure 14. Fits to gel times determined from the maximum in $\tan \delta$ to determine the displayed Arrhenius parameters.

Table 2. Parameters for autocatalytic cure model.

\begin{tabular}{|c|c|c|c|c|c|c|c|}
\hline & & \multicolumn{6}{|c|}{$\begin{array}{c}\text { Autocatalytic: } \mathrm{d} \alpha / \mathrm{d} \boldsymbol{t}=\boldsymbol{k}_{\mathbf{2}} \alpha^{\boldsymbol{m}}(\mathbf{1}-\alpha)^{\boldsymbol{n}} \\
\boldsymbol{k}_{\mathbf{2}} \text { Arrhenius; } \boldsymbol{n}, \boldsymbol{m} \text { given by } \boldsymbol{y}=\mathbf{a} T+\boldsymbol{b} \text { with } \boldsymbol{T} \text { in }{ }^{\circ} \mathbf{C}\end{array}$} \\
\hline & & \multicolumn{2}{|c|}{$k_{2}$} & \multicolumn{2}{|c|}{$m$} & \multicolumn{2}{|c|}{$n$} \\
\hline & & $E_{\mathrm{a}} / R(\mathrm{~K})$ & $\overline{A_{1}\left(\min ^{-1}\right)}$ & a & $b$ & a & $b$ \\
\hline \multirow[t]{2}{*}{$3501-6$} & DSC & 6010 & 24,300 & 0.00615 & -1.50 & -0.00265 & 1.24 \\
\hline & Rheology & 7530 & $3.14 \times 10^{6}$ & 0.00391 & 0.240 & 0.00406 & 1.07 \\
\hline \multirow[t]{2}{*}{ Bryte } & DSC & 9260 & $4.72 \times 10^{9}$ & -0.00276 & 1.00 & 0.0115 & -0.0289 \\
\hline & Rheology & 4350 & 14,400 & -0.00393 & 1.22 & -0.00399 & 2.05 \\
\hline \multirow[t]{2}{*}{ Newport } & DSC & 10,300 & $1.02 \times 10^{11}$ & 0 & 0.742 & 0.0230 & -1.36 \\
\hline & Rheology & 3060 & 662 & -0.00270 & 1.15 & -0.0269 & 4.74 \\
\hline
\end{tabular}


history, which is the same assumption necessary to model extent of reaction with calorimetry. This assumption is probably good for mechanical conversion except near the end of the reaction where $T_{\mathrm{g}}$ becomes similar to the curing temperature; changing temperature and crossing the $T_{\mathrm{g}}$ boundary cause a significant change in modulus with no real change in fractional conversion.

\section{CONCLUSIONS}

Parameters required for predicting the storage modulus $G^{\prime}$ and the relative fractional conversion $\alpha_{\mathrm{r}}$ for three different commercial epoxy resins have been determined. The latter can be modeled using an autocatalytic expression using an experimentally measured relative fractional conversion. Implied in this argument is that some precuring, as well as perhaps not fully reaching $100 \%$ cure at infinite time, does not change the kinetic parameters greatly. A third-order polynomial is proposed for $G^{\prime}$, and a predictive model requires the constant term to be fitted during actual data collection for an arbitrary cure cycle.

A number of other interesting observations were made. As expected, the kinectic parameters measured calorimetrically and rheologically were quantitatively unrelated to one another. The eventual $G_{\max }^{\prime}$ reached at a given isothermal curing temperature did not depend on the temperature below a critical temperature. Above the critical temperature, the modulus dropped swiftly, due to the fact that the $T_{\mathrm{g}}$ was lower than the curing temperature. Finally, the $T_{\mathrm{g}}$ did not correlate to $G_{\max }^{\prime}$ or $H_{\infty}$ at a given temperature over the wide range of curing temperatures studied.

\section{ACKNOWLEDGMENTS}

The financial support for this work was provided by Tec-Masters, Inc. (TMI) under Contract GS-35F-5218H as part of Center for Aircraft and Support Infrastructure (CASI) Research. Henry Pawloski of Alpha Technologies is acknowledged for pointing out an error in this article.

\section{REFERENCES}

1. Ramis, X., Cadenato, A., Morancho, J.M. and Salla, J.M. (2003). Curing of a Thermosetting Powder Coating by Means of DMTA, TMA and DSC, Polymer, 44(7): 2067-2079.

2. Malkin, A.Ya., Gorbunova, I.Yu. and Kerber, M.L. (2005). Comparison of Four Methods for Monitoring the Kinetics of Curing of a Phenolic Resin, Polymer Engineering and Science, 45(1): 95-104.

3. Meyvis, T.K.L., Stubbe, B.G., Van Steenbergen, M.J., Hennink, W.E., De Smedt, S.C. and Demeester, J. (2002). A Comparison Between the Use of Dynamic Mechanical Analysis and Oscillatory Shear Rheometry for the Characterization of Hydrogels, International Journal of Pharmaceuticals, 244(1-2): 163-168.

4. Kamal, M.R. and Sourour, S. (1973). Kinetics and Thermal Characterization of Thermoset Cure, Polymer Engineering and Science, 13(1): 59-64.

5. Ivankovic, M., Incarnato, L., Kenny, J.M. and Nicolais, L. (2003). Curing Kinetics and Chemorheology of Epoxy/Anhydride System, Journal of Applied Polymer Science, 90(11): 3012-3019. 
6. Kim, J., Moon, T.J. and Howell, J.R. (2002). Cure Kinetic Model, Heat of Reaction, and Glass Transition Temperature of AS4/3501-6 Graphite-Epoxy Prepregs, Journal of Composite Materials, 36(21): 2479-2498.

7. Lee, W.I., Loos, A.C. and Springer, G.S. (1982). Heat of Reaction, Degree of Cure and Viscosity of Hercules 3501-6 Resin, Journal of Composite Materials, 16(Nov): 510-520.

8. Ng, S.J., Boswell, R., Claus, S.J., Arnold, F. and Vizzini, A. (2002). Degree of Cure, Heat of Reaction and Viscosity of 8552 and 977-3 HM Epoxy Resins, Journal of Advanced Materials, 34(2): 33-37.

9. See, H. and Nguyen, P. (2004). Using Oscillatory Squeeze Flow to Monitor the Change in Viscoelastic Properties of Curing Materials, Journal of the Society of Rheology, Japan, 32(1): 33-39.

10. Walberer, J.A. and McHugh, A.J. (2001). The Linear Viscoelastic Behavior of Highly Filled Polydimethylsiloxane Measured in Shear and Compression, J. Rheol., 45(1): 187-201.

11. Hou, T.H. and Bai, J.M. (1988). A Semi-Empirical Approach for the Chemoviscosity Modeling of Reactive Resin Systems, Sampe Journal, 24(6): 43-51.

12. Chern, B.C., Moon, T.J., Howell, J.R. and Tan, W. (2002). New Experimental Data for Enthalpy of Reaction and Temperature- and Degree-of Cure-Dependent Specific Heat and Thermal Conductivity of the Hercules 3501-6 Epoxy System, Journal of Composite Materials, 36(17): 2061-2072.

13. White, S.R. and Kim, C. (1993). A Simultaneous Lay-Up and in-situ Cure Process for Thick Composites, Journal of Reinforced Plastics and Composites, 12(5): 520-535.

14. Montserrat, S. and Martin, J.G. (2002). The Isothermal Curing of a Diepoxide-Cycloaliphatic Diamine Resin by Temperature Modulated Differential Scanning Calorimetry, Journal of Applied Polymer Science, 85(6): 1263-1276.

15. Montserrat, S., Andreu, G., Cortes, P., Calventus, Y., Hutchinson, J.M. and Malek, J.J. (1996). Addition of a Reactive Diluent to a Catalyzed Epoxy-Anhydride System. 1. Influence on the Cure Kinetics, Journal of Applied Polymer Science, 61(10): 1663-1674.

16. O'Brien, D.J. and White, S.R. (2003). Cure Kinetics, Gelation, and Glass Transition of a Bisphenol F Epoxide, Polymer Engineering and Science, 43(4): 863-877.

17. Han, C.D. and Lem, K.W. (1984). Chemorheology of Thermosetting Resins 4. The Chemorheology and Curing Kinetics of Vinyl Ester Resin, Journal of Applied Polymer Science, 29(5): 1879-1902.

18. Winter, H.H. and Mours, M. (1997). Rheology of Polymers Near Liquid-Solid Transitions, Advances in Polymer Science, 134: 165-234.

\section{BIOGRAPHIES}

\section{Mitch Hargis}

Mitch Hargis is currently a master's candidate in the School of Chemical, Biological and Materials Engineering at the University of Oklahoma.

\section{Brian Grady}

Brian Grady is a professor in the School of Chemical, Biological and Materials Engineering at the University of Oklahoma. 


\section{Levent Aktas}

Levent Aktas is currently a $\mathrm{PhD}$ candidate in the School of Aerospace and Mechanical Engineering at the University of Oklahoma.

\section{Krishnasumanth R. Bomireddy}

Krishnasumanth R. Bomireddy is currently a master's candidate in the School of Aerospace and Mechanical Engineering at the University of Oklahoma.

\section{Steve Howsom}

Steve Howsom is currently a master's candidate in the School of Aerospace and Mechanical Engineering at the University of Oklahoma.

\section{Cengiz Altan}

Cengiz Altan is a professor in the School of Aerospace and Mechanical Engineering at the University of Oklahoma.

\section{Tom Rose}

Tom Rose is a chief scientist at the Advanced Processing Technology (AvPro) located in Norman, Oklahoma. Tom has over 30 years of experience in the composite industry.

\section{Hans Rose}

Hans Rose is a staff scientist at the Advanced Processing Technology (AvPro) located in Norman, Oklahoma. Hans has over 10 years of experience in the composite industry. 